\title{
Projection effects in coronal mass ejections
}

\author{
B. Vršnak, D. Sudar, D. Ruždjak, and T. Žic
}

Hvar Observatory, Faculty of Geodesy, Kačićeva 26, 10000 Zagreb, Croatia

e-mail: bvrsnak@geof.hr

Received 26 January 2007 / Accepted 16 March 2007

\begin{abstract}
Context. Basic observational parameters of a coronal mass ejection (CME) are its speed and angular width. Measurements of the CME speed and angular width are severely influenced by projection effects.

Aims. The goal of this paper is to investigate a statistical relationship between the plane-of-sky speeds of CMEs and the direction of their propagation, hopefully providing an estimate of the true speeds of CMEs.

Methods. We analyze the correlation between the plane-of-sky velocity and the position of the CME source region, employing several non-halo CME samples. The samples are formed applying various restrictions to avoid crosstalk of relevant parameters. For example, we select only CMEs observed to radial distances larger than 10 solar radii; we omit CMEs showing a considerable acceleration in the considered distance range and treat CMEs of different angular widths separately. Finally, we combine these restriction criteria, up to the limits beyond which the statistical significance of the results becomes ambiguous.

Results. A distinct anti-correlation is found between the angular width of CMEs and their source-region position, clearly showing an increasing trend towards the disc center. Similarly, all of the considered subsamples show a correlation between the CME projected speed and the distance of the source region from the disc center. On average, velocities of non-halo limb-CMEs are 1.5-2 times higher than in the case of non-halo CMEs launched from regions located close to the disc center.

Conclusions. Unfortunately, the established empirical relationships provide only a rough estimate of the velocity correction as a function of the source-region location. To a certain degree, the results can be explained in terms of CME cone models, but only after taking crosstalk of various parameters and observational artifacts into account.
\end{abstract}

Key words. Sun: coronal mass ejection (CMEs)

\section{Introduction}

Observations of coronal mass ejections (CMEs) are strongly affected by projection effects (e.g., Burkepile et al. 2004; Schwenn et al. 2005, and references therein). Since single-coronagraph observations provide only the plane-of-sky measurements of the CME postion, the true radial distances, velocities, accelerations, and angular widths are generally not known. Exceptionally, the true velocity and acceleration can be derived when spectrographic measurements are available (e.g., Raymond et al. 2003; Ciavarella et al. 2005, and references therein).

Understanding of projection effects is important for two reasons. Since the observed kinematics, geometry, and morphology of an eruption is severely influenced by the perspective effects, they also place an important limitation on our understanding of physical characteristics of CMEs. Secondly, the projection effects play an important role in the context of the space weather predictions (e.g., Michałek et al. 2003; Burkepile 2004; Xie et al. 2004; Schwenn et al. 2005; Xue et al. 2005; and references therein).

The relationship between the measured and the true height, velocity, and acceleration of a CME depends on the position of the source-region, the shape of the CME, and the offset of the CME motion from the radial direction. There were several attempts to model projection effects by assuming various geometrical forms of CMEs (e.g., Hundhausen et al. 1994; Sheeley et al. 1999; Leblanc et al. 2001; Zhao et al. 2002; Thernisien et al. 2006). However, due to the unknown 3-dimensional morphology of a CME, the model-based corrections are quite ambiguous, especially if applied to case studies. Most often, it is presumed that CMEs move radially, and the cone shape is applied.

Generally, the model-based procedures employed to infer the true CME velocity could be divided into two classes. In the case of non-halo CMEs, when legs of a CME can be clearly identified, one may apply the model correction straightforwardly, just by measuring the kinematics of the farthest element of the CME leading edge (e.g., Goplaswamy et al. 2001; Yeh et al. 2005). On the other hand, in the case of halo and partial-halo CMEs, launched from regions located close to the disc center, the procedure includes measurements over the entire oval shape of the outer rim of the CME (e.g., Xie et al. 2004; Xue et al. 2005), or at least measurements of the two main axes of the ellipse (e.g., Schwenn et al. 2005).

Unfortunately, it turns out that applying the "correction" usually results in an increase in the data scatter, especially in the case of the former procedure (non-halo CMEs). The correlations between various parameters found from raw data get degraded or even smeared out after applying the correction (e.g., Goplaswamy et al. 2001; Moon et al. 2002; Yeh et al. 2005). Probably, the problem lies in the mentioned model-based methods relying on a prescribed geometrical shape of CMEs, whereas the CME shapes depend on a number of factors and most likely differ significantly from one event to another (e.g., Cremades \& Bothmer 2004).

Therefore, in this paper we study projection effects from the empirical point of view. In particular, we analyze the statistical relationship between the mean CME velocity $v_{\mathrm{m}}$ and the planeof-sky distance $\rho$ of the CME source region from the disc center. 
We employ a large CME sample to compare $v_{\mathrm{m}}$ measured at different $\rho$, with speeds of CMEs launched from the close-to-limb regions. Supposing that the latter are not affected by the projection effects, a correction factor may be derived, providing an estimate of the true CME velocity.

In Sect. 2 we present the data set and basic characteristics of the CME samples used in the analysis, to get insight into the crosstalk of different parameters that could affect the results. In Sect. 3 we analyze the $v_{\mathrm{m}}(\rho)$ relationship employing different susbsets of CMEs in trying to avoid the effects of these crosstalks. In Sect. 4 we discuss the outcome in the frame of three CME cone-model options that are specified in the Appendix. Finally, we consider implications of the results and discuss limitations of possible applications.

\section{The data set}

The following analysis employs a sample of CMEs observed in the range 2-30 solar radii by the Large Angle and Spectrometric COronagraph (LASCO; Brueckner et al. 1995) in the period 1996-2005. We utilize measurements compiled in the online LASCO CME Catalog (http://cdaw.gsfc.nasa.gov/CME_list/; Yashiro et al. 2004). Since the information on the CME source region is needed for the analysis of projection effects, the CME sample was complemented by the data concerning soft X-ray (SXR) flares, for which positional information is available (ftp://ftp.ngdc.noaa.gov/STP/SOLAR_DATA/ SOLAR_FLARES/XRAY_FLARES/).

\subsection{Observational parameters}

In associating flares with CMEs we used spatial and temporal criteria described in Vršnak et al. (2005). In the analysis we considered only CMEs whose leading-edge position was measured in at least four instances. For each CME that satisfied this requirement, we used the linear back-extrapolation of its trajectory to the surface of the Sun in order to estimate the "take-off time" $t_{0}$. Then, following the statistical procedure proposed by Dougherty et al. (2002), we inferred that the flares that occurred out of the temporal window $t_{0} \pm 0.04$ days are most likely not associated with a given $\mathrm{CME}(0.04$ days $\approx 1 \mathrm{~h})$. In this way we established a set of CME-flare pairs whose relative timing was indicative of a causal relationship. In the next step we required the position angle of the flare to lie within the position angle interval spanned by the CME. Finally, we excluded all cases where more than one flare was satisfying the described temporal and spatial criterion, as well as the cases where one flare could be attributed to more than one CME.

In this way we established a sample of 1145 flare-CME pairs (hereinafter denoted as the f-sample), for which it can be presumed that the flare was an intrinsic part of the CME process (e.g., Forbes 2000). For these events, we identify the position of the CME source region with the flare position. In the previous study (Vršnak et al. 2005), it was shown that the described matching procedure results in a certain fraction of wrong CME-flare identifications (about $6 \%$ turned out to be back-side CMEs), which however does not affect the statistical results significantly.

In addition, as a "control" sample, we used a set of 211 CMEs associated with disappearing solar filaments (DSF) and eruptive prominences at the limb (EPL) as reported in Solar Geophysical Data in the period 1996-2001. Hereinafter, we call this the p-sample. A part of this sample (104 events), containing only DSF-associated CMEs that were launched from $\rho<1$, was used by Vršnak et al. (2005).

The CME kinematical parameters are determined from measurements of the plane-of-sky heliocentric distances of the leading edge of CMEs, $R(t)$, expressed in units of the solar radius, $R=r / r_{\odot}$. We employed the following parameters available in the LASCO CME Catalog:

$-v_{\mathrm{m}}-$ mean velocity determined by the linear least-square fit to the $R(t)$ data;

- $a$ - mean acceleration evaluated from the 2 nd-degree polynomial fit to the $R(t)$ data;

- $R_{\mathrm{e}}$ - farthest measured radial distance;

- $W$ - angular width of CME measured at the height beyond which it remains roughly constant.

The CME source-region position can be described either by the angular distance of the CME source region from the solar disk center, $\alpha$, or by the corresponding plane-of-sky radial distance, $\rho=\sin \alpha$. We prefer to use $\rho$, to ease comparison with the values based on the CME cone-models described in the Appendix.

\subsection{Basic characteristics of the sample}

In Fig. 1 we show several basic characteristics of the employed sample that are relevant for the following analysis and the interpretation of results. In Fig. 1a the correlation between the CME mean velocity $v_{\mathrm{m}}$ and the angular width $W$ is presented. We find a distinct correlation, showing that wider CMEs are on average faster (for a similar relationship see, e.g., Hundhausen et al. 1994; Yashiro et al. 2004; Vršnak et al. 2004, 2005). Average values are $\bar{W}=120^{\circ} \pm 110^{\circ}$ and $\bar{v}_{\mathrm{m}}=600 \pm 390 \mathrm{~km} \mathrm{~s}^{-1}$. If halo CMEs are excluded, we find $\bar{W}=84^{\circ} \pm 51^{\circ}$ and $\bar{v}_{\mathrm{m}}=$ $510 \pm 270 \mathrm{~km} \mathrm{~s}^{-1}$.

Figure $1 \mathrm{~b}$ shows the correlation between the radial distance up to which a CME was traced, $R_{\mathrm{e}}$, and the CME velocity. On average, faster CMEs can be traced to greater heights. The distribution of data points also illustrates that halo CMEs tend to be faster than non-halo CMEs (check also Fig. 1a).

The dependence of angular width on the source-region position is presented in Fig. 1c. The correlation reveals that CMEs launched from locations closer to the disc center tend to have larger angular widths, as expected from the CME cone-model (e.g., Yeh et al. 2005). It should be also noted that there is a considerable number of data points representing halo CMEs close to the $\operatorname{limb}\left(W=360^{\circ}\right.$ at $\left.\rho \approx 1\right)$. It is quite likely that at least some of these events were back-side CMEs, erroneously associated with some limb flares (note that spatial criterion defined in Sect. 2 is meaningless in the case of halo CMEs).

In Fig. 1d we show the relationship between the CME velocity and the SXR importance (peak flux) of the associated flare. The correlation shows that CMEs associated with stronger flares are on average faster (see also, e.g., Moon et al. 2002; Burkepile et al. 2004; Vršnak et al. 2005).

The CME data do not show any correlation between the farthest distance $R_{\mathrm{e}}$ and $\rho$, or between the acceleration $a$ and $\rho$. Correlations very similar to that shown in Figs. 1a-d are also found for the p-sample.

\section{Results}

The complete sample of 1145 CMEs does not show any correlation between the position $\rho$ and the CME velocity $v_{\mathrm{m}}$, as would 

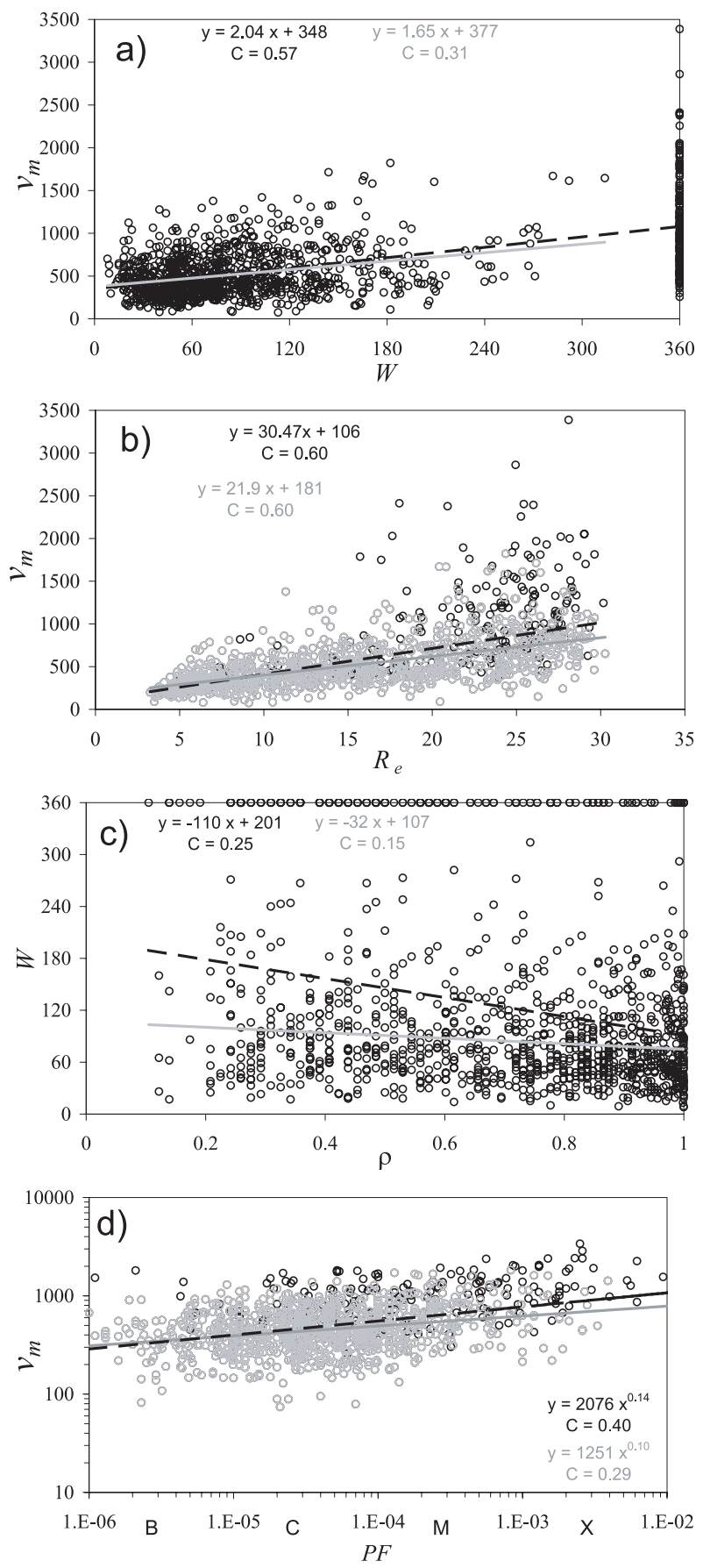

Fig. 1. Relationship between various parameters in the complete f-sample: a) mean velocity and angular width; b) mean velocity and largest measured distance; c) angular width and the distance of the source region from the disc center; d) mean velocity and the 1-8 A peak flux of the associated SXR flare. Black circles represent halo-CMEs. Linear least-square fit parameters are given in the insets (black - complete sample, gray - non-halo CMEs, $C$ is the correlation coefficient). The statistical significance of all correlations is greater than $99.99 \%$.

be expected considering projection effects (see Appendix). In this section we analyze the $v_{\mathrm{m}}(\rho)$ relationship in more detail, employing several subsamples that were formed by successively applying more restrictive conditions to those CME parameters that presumably might affect the outcome. For example, first we exclude halo CMEs. We focus on non-halo CMEs for two reasons: $i$ ) the chance of an erroneous flare-CME association is greater
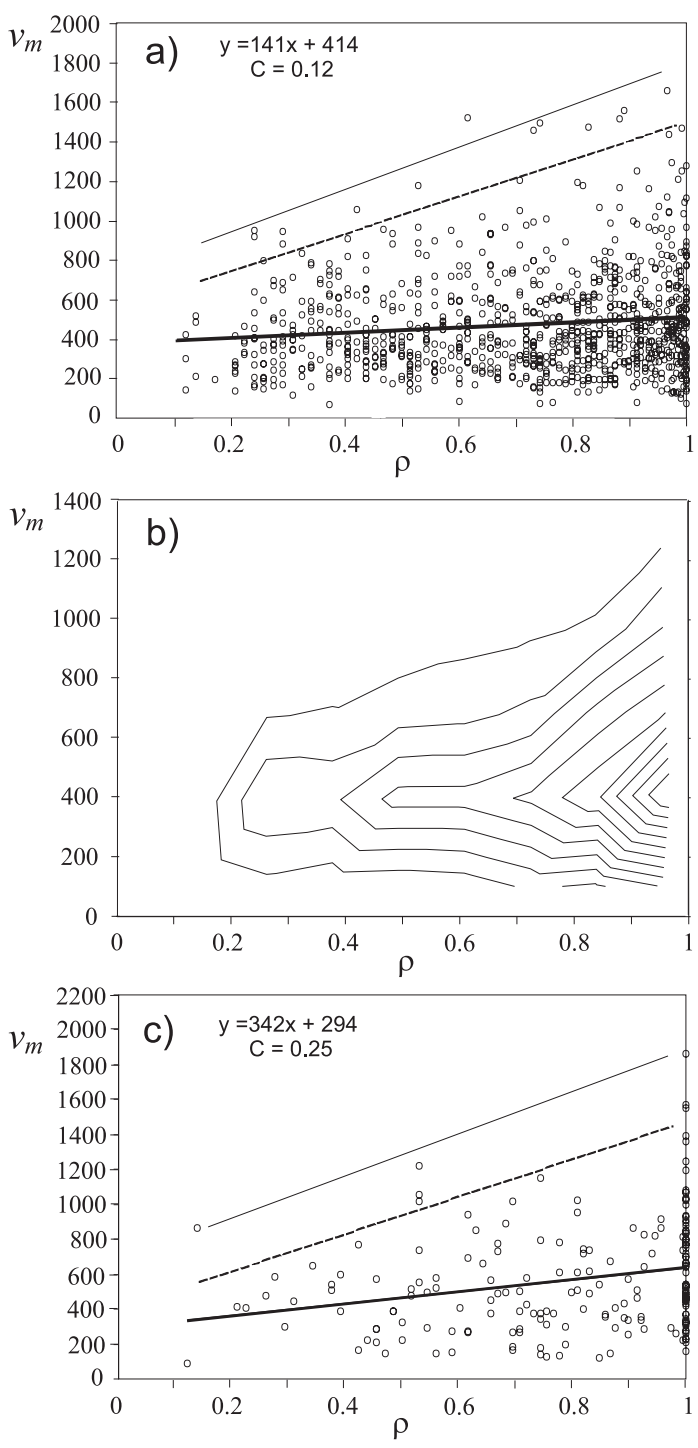

Fig. 2. a) CME mean velocities $v_{\mathrm{m}}$ versus the source-region position $\rho$, shown for the complete non-halo f-sample. b) Contour-plot of the 2-dimensional distribution of the data-points displayed in a). c) Mean velocities versus $\rho$ for the complete non-halo p-sample. The parameters of the linear least-square fit (bold-black lines) in a) and c) are shown in the insets. The thin and dashed lines outline the decreasing trend of the velocity range towards the disc center.

than in normal CMEs (the spatial criterion is ill defined); $i$ ) halo CMEs propagate along the line-of-sight so we observe the expansion velocity rather than the radial one. The latter effect requires a completely different approach to the problem (e.g., Zhao et al. 2002; Michałek et al. 2003; Xie et al. 2004; Xue et al. 2005; Schwenn et al. 2005, and references therein).

\subsection{Complete non-halo CME samples}

In Fig. 2a we show the correlation $v_{\mathrm{m}}(\rho)$ for the complete non-halo f-sample by including only the events with $W<$ $360^{\circ}$. Although the correlation is weak (small correlation coefficients $C$ ), it has a high statistical significance of $P>99.98 \%$; i.e., the correlation is certainly not accidental, since the significance of $P>99.98 \%$ means that the probability of a random distribution of data points (i.e., no correlation) is lower than $0.02 \%$. 
The large scatter (and thus the low correlation coefficient) is a direct consequence of the broad range of true CME velocities. Considering only CMEs that occurred close to the solar limb, we find that true velocities range from 100 to more than $1500 \mathrm{~km} \mathrm{~s}^{-1}$. Checking the velocity range by moving towards the disc center, we see that the $v_{\mathrm{m}}$ range decreases to approximately $100-1000 \mathrm{~km} \mathrm{~s}^{-1}$ at low values of $\rho$. This trend is roughly outlined in Fig. 2a.

It is also important to note that the lower limit of $v_{\mathrm{m}}$ values in Fig. 2a remains around $100 \mathrm{~km} \mathrm{~s}^{-1}$ over the whole $0 \leq \rho \leq 1$ range. This is most likely an observational artifact; i.e., the events with $v_{\mathrm{m}}<100 \mathrm{~km} \mathrm{~s}^{-1}$ are rarely reported as CMEs. Another possible reason is that low-velocity CMEs that are launched from regions located close to the disc center, need a long time/distance before they appear above the coronagraph occulting disc, and quite likely fade out before that. The effect of a constant lower limit over the whole $\rho$ range decreases the slope of the regression line and further reduces the correlation coefficient.

To estimate how much the broad range of velocities and the constant lower limit influence the correlation, we prepared an artificial sample of CMEs, consisting of 15 CME subsets characterized by the true velocities $v^{*}=100,200,300, \ldots, 1500 \mathrm{~km} \mathrm{~s}^{-1}$, each containing $6 \mathrm{CMEs}$ launched from $\rho=0,0.2,0.4,0.6,0.8$, and 1 . Furthermore, we considered that the observed velocities behave as $v=k \rho+v_{0}$, where we took $k=500 \mathrm{~km} \mathrm{~s}^{-1}$. Note that $v_{0}=v^{*}-k$, since $v^{*}=v$ at $\rho=1$. After joining all subsets and applying the lower limit of observed velocities to $100 \mathrm{~km} \mathrm{~s}^{-1}$, the correlation coefficient decreased to $C \approx 0.1$ (for a subset of given $v^{*}$ it amounts to $C=1$ ), whereas the slope became around two times flatter than the original slope $k$.

Taking the linear least-square fit parameters from Fig. 2a, one finds that CMEs launched from the close vicinity of the solar disc center are on average slower by a factor $\kappa_{0} \approx 1.34$ than those launched from the limb regions. We can compare this value with the values derived from the three cone-model options described in the Appendix. The sample shown in Fig. 2a is characterized by the average angular width of $\bar{W}=84^{\circ}$, and for such a width the cone models $\mathrm{A}, \mathrm{B}$, and $\mathrm{C}$ described in the Appendix, give values $\kappa_{0}=1.5,2$, and 2.5, respectively. Whereas the model $\mathrm{A}$ value might be considered as comparable to the observed one, the values based on models $\mathrm{B}$ and $\mathrm{C}$ are considerably higher. Even taking into account the decreased slope of the correlation due to the previously mentioned effects, one finds the ratio $\kappa_{0}$ is not higher than $\approx 1.7$.

On the other hand, focusing on the thin or dashed line in Fig. 2a, which roughly outline the decreasing trend of the $v_{\mathrm{m}}$ range towards the disc center (hereinafter "upper limit" trend), we find a considerably higher values of $\kappa_{0}$, ranging between 2 and 3. Such values are roughly in the range expected for the cone models $\mathrm{B}$ and $\mathrm{C}$.

To define the decreasing trend in the $v_{\mathrm{m}}$ range in a more quantitative way, we present the 2-dimensional distribution in Fig. 2b of the data-points from Fig. 2a. The first isoline shows a trend from some $1000-1200 \mathrm{~km} \mathrm{~s}^{-1}$ at $\rho=1$ down to about $500 \mathrm{~km} \mathrm{~s}^{-1}$ at $\rho=0$. This corresponds to the ratio $\kappa_{0} \approx 2-2.4$; i.e., very close to the values derived from the cone models $\mathrm{B}$ and $\mathrm{C}$.

In Fig. $2 \mathrm{c}$ we display the $v_{\mathrm{m}}(\rho)$ correlation for the complete non-halo p-sample. Utilizing the linear least-square fit parameters, we find $\kappa_{0} \approx 2.16$. The mean angular width of CMEs in the non-halo p-sample equals to $\bar{W}=88^{\circ}$, and for such a width models A, B, and C give $\kappa_{0}=1.4,2.0$, and 2.4, respectively. The model $\mathrm{B}$ and $\mathrm{C}$ values are close to the measured ones, whereas
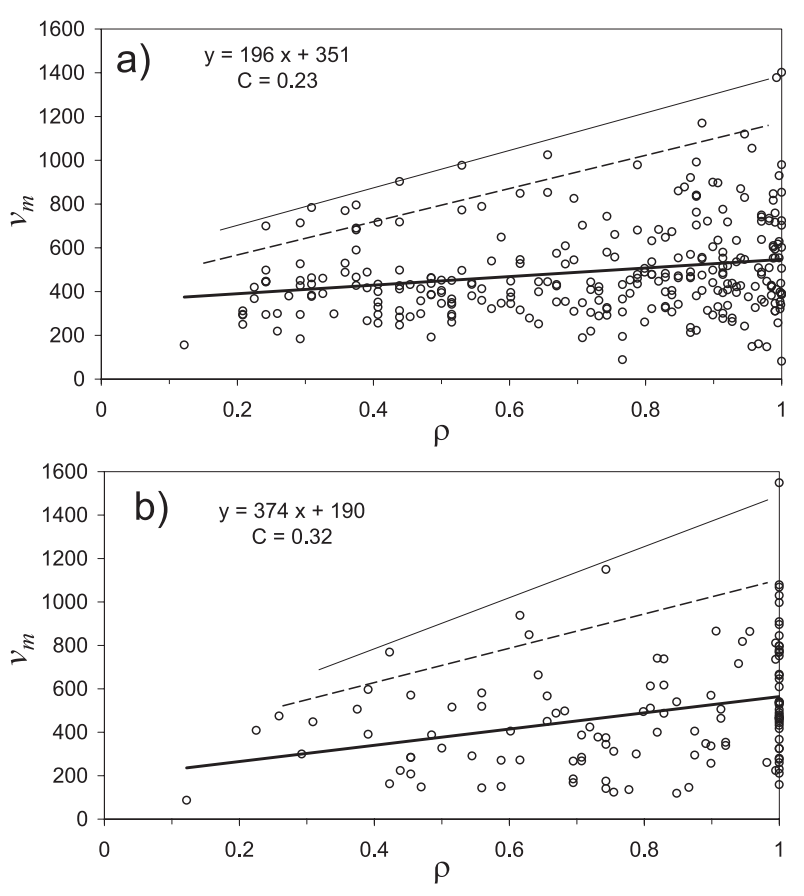

Fig. 3. The correlation between mean velocities and the source-region position for: a) non-halo f-sample restricted to $|a|<5 \mathrm{~m} \mathrm{~s}^{-2}$ and $R_{\mathrm{e}}>$ 10 ; b) non-halo p-sample restricted to $|a|<10 \mathrm{~m} \mathrm{~s}^{-2}$. The least-square fit parameters are given in the insets. Statistical significances are $P>$ 99.95\% and $P>99.97 \%$, respectively. Bold lines represent the linear least-square fit, whereas thin and dashed lines outline the "upper limit" trend like in Figs. 2a and 2c.

model A gives considerably lower values. Again, we outline the decreasing trend of the $v_{\mathrm{m}}$ range. The values of $\kappa_{0}$ based on these lines are found to be around 3 ; i.e., somewhat higher than the model values.

\subsection{Reduced samples}

Bearing in mind the relationships presented in Fig. 1 and the corresponding crosstalk of parameters, we reduce our f-sample by excluding events with $R_{\mathrm{e}}<10$. Furthermore, to exclude the effects of acceleration, we consider only events with $|a|<5 \mathrm{~m} \mathrm{~s}^{-2}$ : events launched at small $\rho$ are observed in the late phase of the eruption, when the main acceleration is over, whereas a significant part of CMEs still accelerates in the case of limb events (e.g., Feynman \& Ruzmaikin 2004; Vršnak et al. 2005). Finally, we subdivide the sample into $W$-bins, since it is expected that the projection effects depend on the CME geometry (see Appendix).

Successively applying a more restrictive acceleration limit; i.e., decreasing the limiting value from, e.g., $|a|<20$ to $|a|<$ $5 \mathrm{~m} \mathrm{~s}^{-2}$, we found that the slope of the $v_{\mathrm{m}}(\rho)$ relationship is systematically increasing. A similar effect we find also if we successively increase the limiting value of $R_{\mathrm{e}}$. In Fig. 3a we show the non-halo f-sample, including only the events with $|a|<5 \mathrm{~m} \mathrm{~s}^{-2}$, $R_{\mathrm{e}}>10$. We see that the slope and the correlation coefficient are larger than in Fig. 2a. Taking the parameters of the linear leastsquare fit, we find that the ratio of $v_{\mathrm{m}}$ at $\rho=1$ and $\rho=0$ amounts to $\kappa_{0}=1.56$. This value is relatively close to the values derived from models A and B, adding up to 1.4 and 1.9. On the other hand, it is considerably lower than that obtained from model $\mathrm{C}$, amounting to 2.3. Considering the "upper limit" trend, $\kappa_{0}$ ranges between 2 an 2.5 . These values are quite close to what is 
Table 1. Characteristics of the correlation $v_{\mathrm{m}}=k \rho+v_{0}$, specified for the non-halo CME samples displayed in Figs. 2-4. The values $\kappa_{A}, \kappa_{B}$, and $\kappa_{C}$ displayed in the last column represent the values of $\kappa_{0}$ derived from models $\mathrm{A}, \mathrm{B}$, and $\mathrm{C}$, respectively.

\begin{tabular}{|c|c|c|c|c|c|c|c|c|c|c|}
\hline Sample & Fig. & $W$-bin & $N$ & $\bar{W}$ & $k$ & $v_{0}$ & F-test $(\%)$ & C & $\kappa_{0}$ & $\kappa_{A} / \kappa_{B} / \kappa_{C}$ \\
\hline f-sample & $2 a$ & $W<360^{\circ}$ & 979 & $84^{\circ} \pm 51^{\circ}$ & $141 \pm 37$ & $414 \pm 28$ & $>99.98$ & 0.12 & 1.34 & $1.5 / 2.0 / 2.5$ \\
\hline p-sample & $2 \mathrm{c}$ & $W<360^{\circ}$ & 197 & $88^{\circ} \pm 52^{\circ}$ & $342 \pm 95$ & $294 \pm 79$ & $>99.95$ & 0.25 & 2.16 & $1.4 / 2.0 / 2.4$ \\
\hline f-sample & $3 \mathrm{a}$ & $W<360^{\circ}$ & 289 & $96^{\circ} \pm 51^{\circ}$ & $196 \pm 50$ & $351 \pm 38$ & $>99.99$ & 0.23 & 1.56 & $1.3 / 1.9 / 2.3$ \\
\hline p-sample & $3 b$ & $W<360^{\circ}$ & 123 & $90^{\circ} \pm 50^{\circ}$ & $394 \pm 100$ & $190 \pm 83$ & $>99.97$ & 0.32 & 2.97 & $1.4 / 2.0 / 2.4$ \\
\hline f-sample & $4 a$ & $30^{\circ} \leq W<60^{\circ}$ & 89 & $46^{\circ} \pm 8^{\circ}$ & $190 \pm 78$ & $251 \pm 63$ & $>98$ & 0.25 & 1.76 & $2.6 / 3.4 / 3.6$ \\
\hline f-sample & $4 a$ & $60^{\circ} \leq W<90^{\circ}$ & 92 & $72^{\circ} \pm 9^{\circ}$ & $146 \pm 98$ & $345 \pm 78$ & $>85$ & 0.15 & 1.42 & $1.7 / 2.4 / 2.7$ \\
\hline f-sample & $4 a$ & $90^{\circ} \leq W<120^{\circ}$ & 68 & $102^{\circ} \pm 9^{\circ}$ & $150 \pm 110$ & $328 \pm 77$ & $>82$ & 0.16 & 1.45 & $1.3 / 1.8 / 2.3$ \\
\hline p-sample & $4 b$ & $W<60^{\circ}$ & 25 & $42^{\circ} \pm 10^{\circ}$ & $443 \pm 197$ & $143 \pm 167$ & $>94$ & 0.43 & 4.10 & $2.8 / 3.6 / 3.8$ \\
\hline p-sample & $4 b$ & $60^{\circ} \leq W<120^{\circ}$ & 43 & $87^{\circ} \pm 15^{\circ}$ & $138 \pm 140$ & $378 \pm 117$ & $>67$ & 0.15 & 1.37 & $1.5 / 2.1 / 2.5$ \\
\hline
\end{tabular}

expected from cone models $\mathrm{B}$ and $\mathrm{C}$, which give the ratios of 1.9 and 2.3 , respectively.

Analogously, in Fig. $3 \mathrm{~b}$ we show the non-halo p-sample; however, since the p-sample is considerably smaller, we could only take the less restrictive condition $|a|<10 \mathrm{~m} \mathrm{~s}^{-2}$ (instead of $|a|<5 \mathrm{~m} \mathrm{~s}^{-2}$ ). The linear least-square fit gives the value of $\kappa_{0}=2.97$, which is considerably higher than the values based on models $\mathrm{A}, \mathrm{B}$, and $\mathrm{C}$, which for $\bar{W}=90^{\circ}$ equal to $1.4,2.0$, and 2.4, respectively. Again, we indicate the "upper limit" trend in Fig. $3 \mathrm{~b}$, from which we find $\kappa_{0}$ ranging between 3 and 5 . These values are much higher than the model values, but it should be noted that the "upper limit" lines in this case are based on quite a small number of data points (especially the dotted one).

\subsection{Width bins}

Since the projection effect on $v_{\mathrm{m}}$ is likely to be stronger for CMEs of smaller widths (see Appendix), we subdivide the sample presented in Fig. 2 a into three $W$-bins: $30^{\circ} \leq W<60^{\circ}$, $60^{\circ} \leq W<90^{\circ}, 90^{\circ} \leq W<120^{\circ}$ (the bin $W<30^{\circ}$ includes a small number of events, and the results are statistically insignificant). The results are shown in Fig. 4a. Applying still more stringent conditions or narrowing $W$-bins reduces the samples too much, so the correlations become statistically insignificant. The three considered subsamples consist of $N=89,92$, and 68 events. The mean widths are $46^{\circ}, 72^{\circ}$, and $102^{\circ}$. Figure $4 \mathrm{a}$ shows that the bin $30^{\circ} \leq W<60^{\circ}$ displays a steeper slope than the last two, but the last two are very similar. The ratios $\kappa_{0}$ amount to $1.76,1.42$, and 1.45 for the three subsets, respectively, and it should be noted that the differences are of low statistical significance. The values for the two narrowest bins are considerably lower than the model values, whereas $\kappa_{0}$ for the widest bin is close to the model A value (compare the last two columns of Table 1 where we present the observed and the model-based values).

In Fig. 4b we show results for the p-sample taking only the CMEs with $|a|<10 \mathrm{~m} \mathrm{~s}^{-2}$ and dividing them into $\Delta W=60^{\circ}$ bins. The two considered subsamples consist of 41 and 50 events. The mean widths are $42^{\circ}$ and $85^{\circ}$. The ratio $\kappa_{0}$ equals 4.10 and 1.37 , respectively. The former value is somewhat higher than the model values, whereas the latter one is comparable with the model A value (see the last column of Table 1). The difference between the two subsets is within the accuracy of the fit parameters; i.e., it again has low statistical significance.
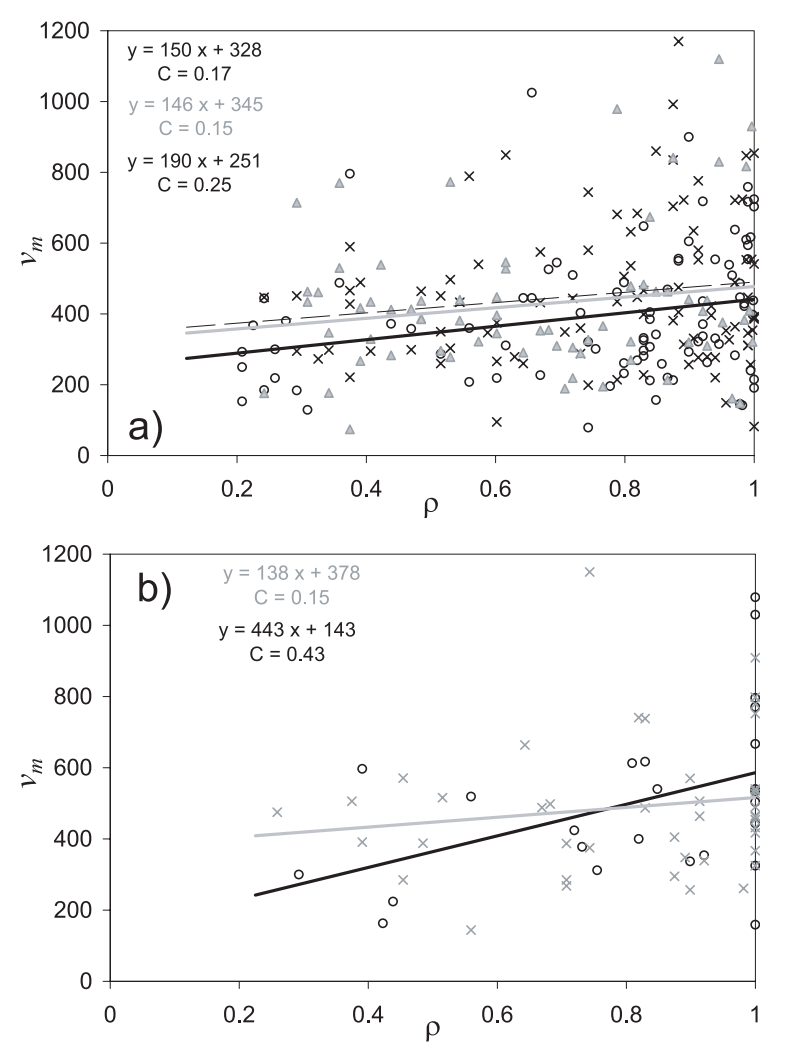

Fig. 4. The correlation between mean velocities and the source-region position for the reduced non-halo CME samples shown in Fig. 3, divided in $W$-bin subsamples: a) f-sample $\left(30^{\circ} \leq W<60^{\circ}\right.$ - black dots and black-thick line, $60^{\circ} \leq W<90^{\circ}-$ gray triangles and gray-thick line, $90^{\circ} \leq W<120^{\circ}$ - crosses and gray-dashed line); b) the p-sample $\left(W<60^{\circ}\right.$ - black, $60^{\circ} \leq W<120^{\circ}-$ gray). The least-square fit parameters are given in the insets.

Bearing Fig. 1d in mind, we also checked the correlations by employing only events associated with SXR C-class flares (the most abundant subset). It turned out that the outcome is more or less the same as when not applying the SXR-class restriction; i.e., differences are statistically insignificant.

\subsection{Summary of correlations and the empirical correction}

Basic characteristics of the $v_{\mathrm{m}}(\rho)$ correlations presented in Figs. 2-4 are summarized in Table 1. After defining the 
samples in the first three columns, we display the number of events in the sample $N$, and the mean angular width of CMEs in the sample, $\bar{W}$. In the next five columns we present the slope $k$ of the regression line, the $y$-axis intercept $v_{0}$ of the regression line, the statistical F-test significance of the correlation, the correlation coefficient $C$, and the ratio $\kappa_{0}$ of the $v_{\mathrm{m}}$ values at $\rho=1$ and $\rho=0$. In the last column we display the values of $\kappa_{0}$ derived from models A, B, and C, denoted as $\kappa_{A}, \kappa_{B}$, and $\kappa_{C}$, respectively.

Bearing in mind the effects discussed in Sect. 3.1, the values of the factor $\kappa_{0}$ obtained from the linear least-square parameters listed in Table 1 are most likely underestimated; i.e., the correction factor is likely to be larger. Indeed, inspecting Table 1 we find that in most cases the value of $\kappa_{0}$ is lower than the model values.

The parameter $\kappa_{0}$ represents the empirical factor by which one should multiply the velocity of a CME launched from a close-to-center source region in order to get its true velocity. In fact, a correlation between the CME velocities and the sourceregion position, expressed in the form $v_{\mathrm{m}}=k \rho+v_{0}$, can be utilized to obtain factor $\kappa(\rho)$, by which the observed velocity $v$ of a CME that was launched from any position $\rho$ should be multiplied to get its true velocity $v^{*}$ :

$\kappa(\rho)=\frac{k+v_{0}}{\rho k+v_{0}}$.

In Fig. 5a we show the correction factor $\kappa(\rho)$ for the $W$-bin subsets of the f-sample shown in Fig. 4a. In Fig. 5b we show the model functions $\kappa(\rho)$ derived from model B for the appropriate mean widths of CMEs in the three subsets shown in Fig. 5a. Comparing the observation-based and the model-based dependencies, we see that the observation-based values are considerably lower. In Fig. 5c, we directly compare the two width-bin subsets of the p-sample displayed in Fig. $4 \mathrm{~b}$ with the model B values. Here, the narrower bin is close to the model values, but the wider bin is again showing considerably lower observationbased values.

\section{Discussion and conclusion}

We summarize the results of the presented analysis as follows:

1. Our sample of non-halo CMEs shows a weak, but statistically significant anti-correlation between CME angular widths $W$ and the source-region distance from the disc center $\rho$.

2. Similarly, we found a weak, but statistically significant correlation between the velocities $v_{\mathrm{m}}$ of non-halo CME and the source-region position $\rho$.

3. The $v_{\mathrm{m}}(\rho)$ correlation gets better if the sample is reduced by excluding CMEs that show acceleration or dissolve before reaching, e.g., $R_{\mathrm{e}}=10$.

4. The range of $\mathrm{CME}$ velocities successively decreases by restricting $\rho$ to values closer to the disc center.

5. Considering different subsamples, we find that the ratio of velocities of CMEs close to the limb and close to the disc center most often ranges between $\kappa_{0} \approx 1.5$ and 2 .

6 . The data indicate that the ratio $\kappa_{0}$ might be larger for narrower CMEs, as expected from CME cone-models; however, the differences have a very low statistical significance.

The large data scatter, resulting in low correlation coefficients for the $v_{\mathrm{m}}(\rho)$ correlation, is due to the wide range of CME velocities involved. Likewise, the correlation is degraded by the lower limit to $v_{\mathrm{m}}$ values around $100 \mathrm{~km} \mathrm{~s}^{-1}$ over the whole
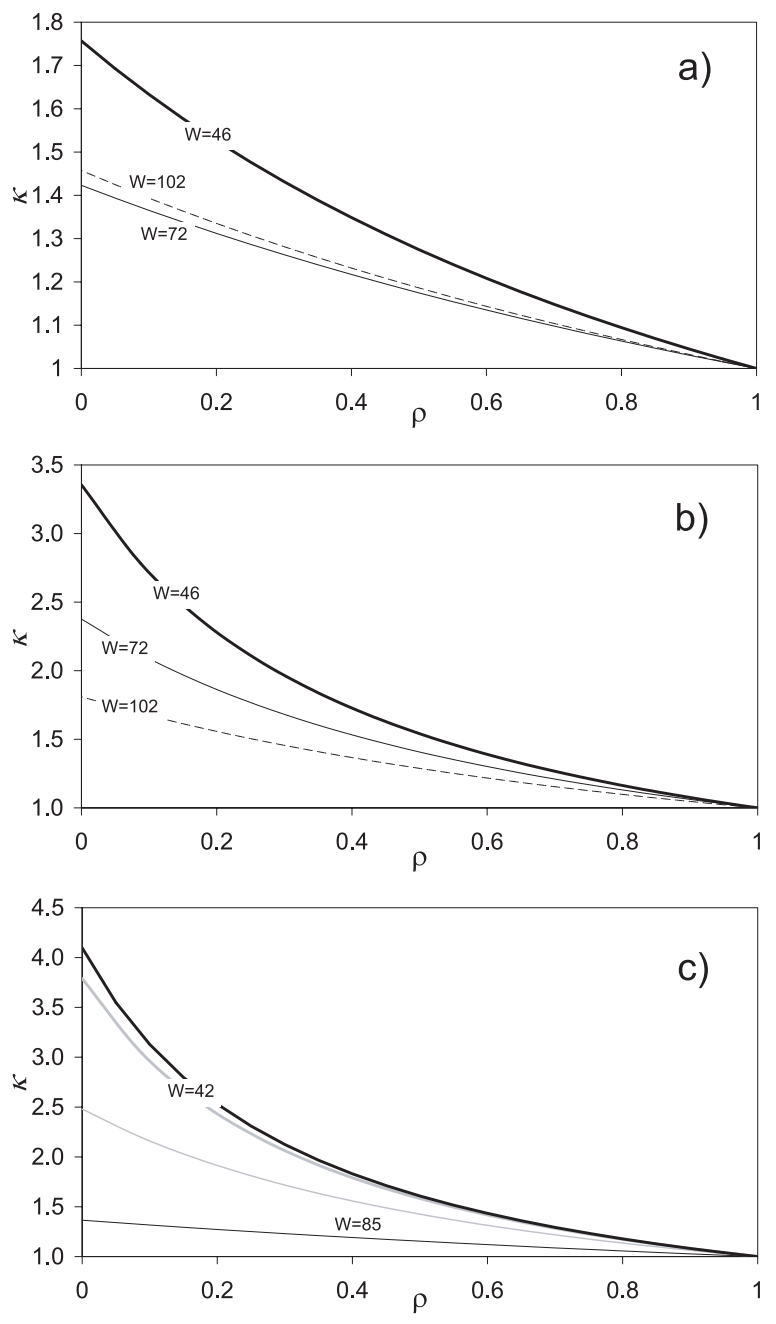

Fig. 5. Comparison of the empirical and the model-based correction factors $\kappa(\rho)$. a) Empirical values based on f-subsamples shown in Fig. 4a $\left(30^{\circ} \leq W<60^{\circ}\right.$ thick, $60^{\circ} \leq W<90^{\circ}$ thin, and $90^{\circ} \leq W<120^{\circ}$ dashed; mean widths are written by the curves). b) Correction factors derived from the cone model B by employing mean widths of CME subsamples shown in a). c) Empirical correction factors based on p-subsamples shown in Fig. $4 \mathrm{~b}\left(W<60^{\circ}\right.$ - black-thick, $60^{\circ} \leq W<$ $120^{\circ}$ - black-thin) compared with the correction factors based on the cone model B calculated for the corresponding mean widths (gray thick and thin lines, respectively).

$0<\rho<1$ range. This is most likely an artifact; i.e., the events with $v_{\mathrm{m}}<100 \mathrm{~km} \mathrm{~s}^{-1}$ are only occasionally reported as CMEs. Another possible reason is that low-velocity CMEs launched from locations close to the disc center need a long time/distance before they appear above the occulting disc, and so quite likely fade out before that. If such events are still to be seen in the LASCO field-of-view, they have to be "intense" events, which are usually faster than average. The nature of Thompson scattering also increases the selection effect, enabling only "intense" events to be seen if launched from regions close to the disc center. Because Thompson scattering is the most effective in the direction perpendicular to the incident light, CMEs are more easily observed when traveling perpendicular to the observer than when heading toward us.

Although the presented analysis reveals a distinct correlation between the CME speed and position, from the obtained results it is difficult to establish an unambiguous empirical relation to be used for deriving a true CME speed from the measured one. The 
values of $\kappa_{0}$ based on the parameters of the linear least-square fit for different subsamples, generally range from 1.5 and 2. However, the $v_{\mathrm{m}}(\rho)$ trend for the fastest events indicates that $\kappa_{0}$ may be higher by a factor of two; i.e., the correction based on the least-square fit should be considered as a lower limit.

It is interesting to note that, although the cone model A should be considered as geometrically less realistic, it shows a better agreement with our results based on the least-square fit procedure than models B and C. On the other hand, models B and $\mathrm{C}$ show a better correspondence with the $v_{\mathrm{m}}(\rho)$ behavior for the fastest events ("upper limit" trend). This again indicates that the correction factor based on the least-square fit procedure is probably underestimated and that the "upper limit" procedure gives more appropriate values. Finally, it should be noted that the CME cone models predict a larger correction factor for narrower CMEs. Although our results indicate such a trend, the differences have very low statistical significance, and the results are not reliable enough to provide a clear $\kappa_{0}(W)$ dependence.

In this respect, it should be noted that the CME cone model is a purely morphological geometric concept, not founded on physical considerations. Thus, it is instructive to compare our results with models that are based on the physics of the eruption. In particular, we pay attention to the geometry included in models, since it is the main factor when projection effects are considered. Unfortunately, this aspect is treated only occasionally and only in an illustrative way (e.g., Tokman \& Bellan 2002; Thernisien et al. 2006; Krall et al. 2006), because most of the models are focused on the dynamics of the eruption.

According to the geometry, magnetohydrodynamical models of CMEs could be classified into "global" models and "local" ones. The first class treats the eruption as a global process, taking place in a large-scale coronal streamer. Consequently, the eruption is most often treated as a 2-dimensional (2-D) or 2.5-D process in the spherical geometry, where the pre-eruptive coronal streamer, either bipolar (e.g., Mikić \& Linker 1994; Cargill \& Schmidt 2002; Jackobs et al. 2006, and references therein) or quadrupolar (e.g., Antiochos et al. 1999), is sited on, or parallel to, the solar equator. Due to the axial symmetry involved, these models are not affected by the projection effects. Since our results show the dependence of the plane-of-sky velocity and the CME width on the source-region position, it can be concluded that situations where such 2-D and 2.5-D axially symmetric models could be applied are relatively rare (large-scale eruptions oriented in the East-West direction and occupying a very wide range of solar longitudes).

The most advanced global models, whose development has begun only recently, start from a realistic magnetohydrodynamical state of the corona and the solar wind, and the eruption only includes a limited magnetic structure in the corona (e.g., Fry et al. 2003; Manchester et al. 2004; Odstrčil et al. 2004; Odstrčil et al. 2005, and references therein). The model results reveal a wide variety of morphological patterns and evolutionary characteristics of eruptions. Unfortunately, the projection effects are never directly treated in this type of model. However, since only a fragment of the corona is included in the eruption, it is natural to expect that the outcome is affected by the projection effects; i.e., that the plane-of-sky velocity and the CME width depend on the source-region position. That can be seen directly from the presentation of model results that often includes various viewing angles to represent the results more transparently. This variety of morphological and evolutionary forms, probably leads to complex projection effects that are much more intricate than expected from the simple cone model.
In contrast to global models, which primarily treat the propagation of CMEs in a realistic ambience, "local" models are focused on the processes causing the take-off and acceleration of the eruptive coronal structure. Most of these models invoke magnetic structures that include semi-toroidal flux-rope embedded in the magnetic arcade and anchored at both ends in the dense photosphere (for the development of the flux-rope concept see, e.g., Anzer 1978; Mouschovias, \& Poland 1978; Chen 1989; Vršnak 1990; Chen \& Krall 2003). The fast progress of numerical techniques have recently enabled a very advanced modeling of such a system, also providing the inclusion of magnetic field reconnection (e.g., Amari et al. 2000; Amari et al. 2003; Roussev et al. 2003; Kliem et al. 2004; Török \& Kliem 2005; Birn et al. 2006; Gibson \& Fan 2006; for a review see Gibson et al. 2006). From the point of view of projection effects, it is important to note that this class of models, based on the magnetic field configuration proposed by Titov \& Demoulin (1999), is not axi-symmetric, so the approximation in terms of the cone geometry is not appropriate. Consequently, the projected width of the CME depends not only on the location of the source region, but also on the orientation of the plane containing the flux-rope axis (see, e.g., Fig. 15 of Cremades \& Bothmer 2004). Similarly, the relationship between the plane-of-sky velocity and the source-region position depends on the flux-rope orientation. Thus, again we find a much more complex situation than in the cone geometry, certainly leading to the degradation of the $W$-bin based correlations $v_{\mathrm{m}}(\rho)$ and, in particular, the $\kappa_{0}(W)$ dependence. Finally, it should be noted that these numerical simulations reveal significant morphological changes caused by kinking of the flux rope axis and by reconnection, additionally degrading the $v_{\mathrm{m}}(\rho)$ correlation.

As a conclusion, it is not surprising that the correlation of the plane-of-sky velocity and the source-region position is much weaker than expected from the simple cone model, since real CMEs appear in a broad variety of types and shapes and are generally much more complex than represented by any of actual models.

Acknowledgements. This work was sponsored by the Air Force Office of Scientific Research, USAF, under grant number FA8655-06-1-3036. We are thankful to the LASCO-SOHO and GOES teams for operating the instruments and performing the basic data reduction. We are especially grateful to Nat Gopalswamy, Seiji Yashiro, Grzegorz Michałek, and their colleagues for compiling the online LASCO CME Catalog.

\section{Appendix: The CME cone models}

The relationship between the plane-of-sky velocity $v$ and the true velocity $v^{*}$ depends on the CME geometry. In this paper we consider three different CME cone models frequently used in CME studies. At the top of the cone model A has a spherical surface concentric with the solar surface, model B has a half-sphere, whereas in model $\mathrm{C}$ a spherical surface is matched tangentially to the cone surface (see, e.g., Fig. 9 of Schwenn et al. 2005). Models A, B, and C relate the plane-of-sky velocity $v$ and the true velocity $v^{*}$ as:

$v=v^{*} \sin \left(\alpha+\phi^{*}\right)$

$v=v^{*} \frac{\sin \alpha+\tan \phi^{*}}{1+\tan \phi^{*}}$

$v=v^{*} \frac{\sin \alpha+\sin \phi^{*}}{1+\sin \phi^{*}}$

respectively, where $\sin \alpha=\rho$, and $\phi^{*}=W^{*} / 2$ is the true halfwidth of the cone related to the observed one as:

$\tan \phi^{*}=\rho \tan \phi$. 

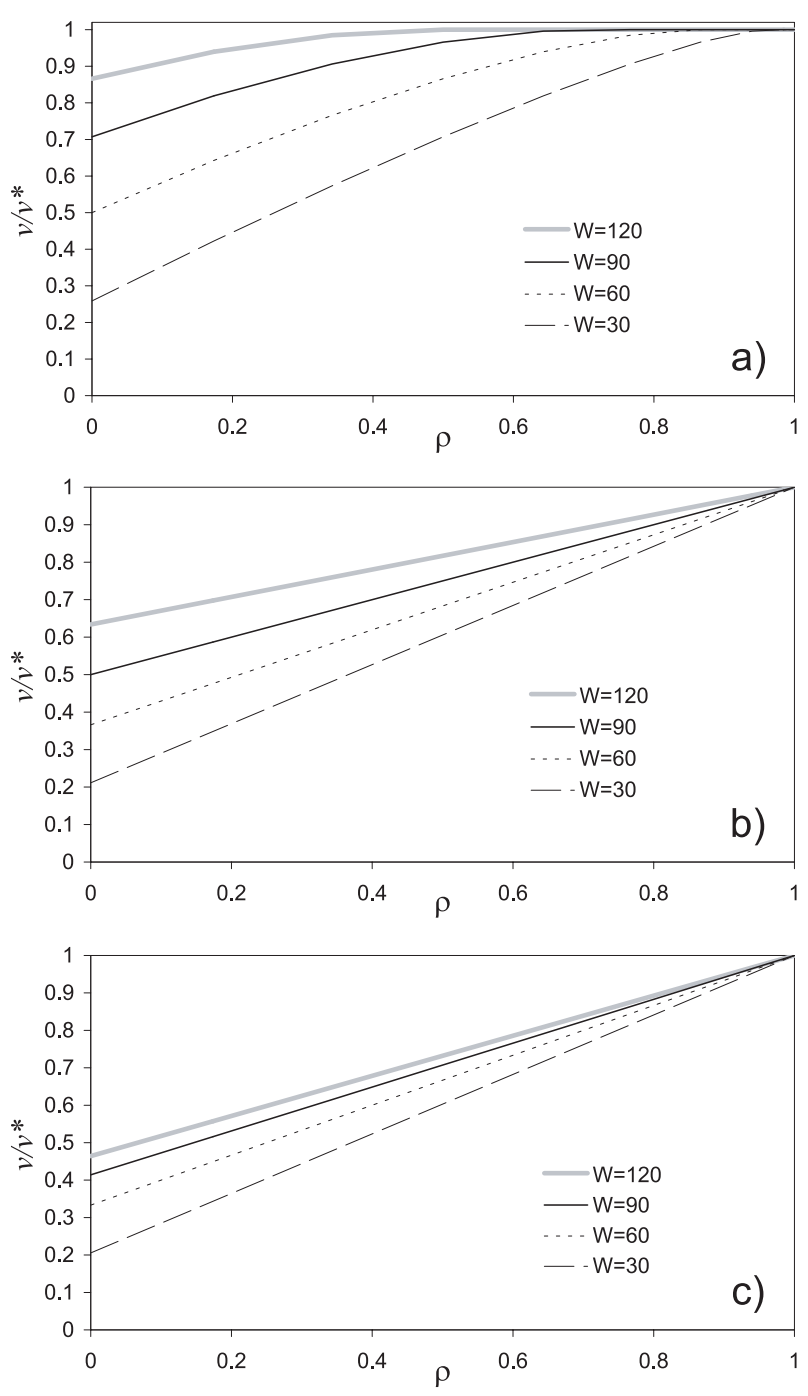

Fig. 6. The ratio of the plane-of-sky velocity $v$ and the true velocity $v^{*}$, presented as a function of the source-region position, derived from a) model $\mathrm{A}, \mathbf{b})$ model $\mathrm{B}, \mathbf{c})$ model $\mathrm{C}$.

In Fig. 6 we show the behavior of the ratio $v / v^{*}=1 / \kappa$. Note that models $\mathrm{B}$ and $\mathrm{C}$ give $v / v^{*}=1$ only at $\rho=1$, whereas in model A the projected velocity becomes equal to the true velocity already at smaller $\rho$, beyond which $v^{*}=v=$ const. Note that the three described models are valid only for $\phi^{*}<90^{\circ}$; i.e., $W^{*}<180^{\circ}$.

Figure 6 shows that the projection effect should be larger for narrower CMEs; i.e., the slopes of $v / v^{*}$ are steeper. The ratio of the true velocity $v^{*}$ and the observed velocity $v$ is highest in model $\mathrm{C}$ and lowest in model $\mathrm{A}$.

\section{References}

Amari, T., Luciani, J. F., Mikić, Z., \& Linker, J. 2000, ApJ, 529, L49 Amari, T., Luciani, J. F., Aly, J. J., Mikić, Z., \& Linker, J. 2003, ApJ, 585, 1073 Antiochos, S. K., DeVore, C. R., \& Klimchuk, J. A. 1999, ApJ, 510, 485 Anzer, U. 1978, Sol. Phys., 57, 111

Birn, J., Forbes, T. G., \& Hesse, M. 2006, ApJ, 645, 732

Brueckner, G. E., Howard, R. A., Koomen, M. J., et al. 1995, Sol. Phys., 162, 357

Burkepile, J. T., Hundhausen, A. J., Stanger, A. L., St. Cyr, O. C., \& Seiden, J. A. 2004, J. Geophys. Res., 109, 3103

Cargill, P. J., \& Schmidt, J. M. 2002, Ann. Geophys., 20, 879

Chen, J. 1989, ApJ, 338, 453

Chen, J., \& Krall, J. 2003, J. Geophys. Res., 108, 1410

Ciaravella, A., Raymond, J. C., Kahler, S. W., Vourlidas, A., \& Li, J. 2005, ApJ, 621,1121

Cremades, H., \& Bothmer, V. 2004, A\&A, 422, 307

Dougherty, B. L., Zirin, H., \& Hsu, K. 2002, ApJ, 577, 457

Feynman, J., \& Ruzmaikin, A. 2004, Sol. Phys., 219, 301

Forbes, T. G. 2000, J. Geophys. Res., 105, 23153

Fry, C. D., Dryer, M., Deehr, C. S., et al. 2003, J. Geophys. Res., 108, 1070

Gibson, S. E., \& Fan, Y. 2006, ApJ, 637, L65

Gibson, S. E., Fan, Y., Török, T., \& Kliem, B. 2006, Space Sci. Rev., 124, 131

Gopalswamy, N., Yashiro, S., Kaiser, M. L., Howard, R. A., \& Bougeret, J.-L. 2001, J. Geophys. Res., 106, 29219

Hundhausen, A. J., Burkepile, J. T., \& St. Syr, O. C. 1994, J. Geophys. Res., 99, 6543

Jacobs, C., Poedts, S., \& van der Holst, B. 2006, A\&A, 450, 793

Kliem, B., Titov, V. S., \& Török, T. 2004, A\&A, 413, L23

Krall, J., Yurchyshyn, V. B., Slinker, S., Skoug, R. M., \& Chen, J. 2006, ApJ, 642,541

Leblanc, Y., Dulk, G. A., Vourlidas, A., \& Bougeret, J.-L. 2001, J. Geophys. Res., 106, 25301

Manchester, W. B., Gombosi, T. I., Russev, I., et al. 2004, J. Geophys. Res., 109

Michałek, G., Gopalswamy, N., \& Yashiro, S. 2003, ApJ, 584, 472

Mikić, Z., \& Linker, J. 1994, ApJ, 430, 898

Moon, Y.-J., Choe, G. S., Wang, H., et al. 2002, ApJ, 581, 694

Mouschovias, T. Ch., \& Poland, A. I. 1978, ApJ, 220, 675

Odstrčil, D., Riley, P., \& Zhao, X. P. 2004, J. Geophys. Res., 109

Odstrčil, D., Pizzo, V. J., \& Arge, C. N. 2005, J. Geophys. Res., 110

Raymond, J. C., Ciaravella, A., Dobrzycka, D., et al. 2003, ApJ, 597, 1106

Roša, D., Vršnak, B., Božić, H., et al. 1998, Sol. Phys., 179, 237

Roussev, I. I., Forbes, T. G., Gombosi, T. I., et al. 2003, ApJ, 588, L45

Schwenn, R., Dal Lago, A., Huttunen, E., \& Gonzalez, W. D. 2005, Ann. Geophys., 23, 1033

Sheeley Jr., N. R., Howard, R. A., Koomen, M. J., \& Michels, D. J. 1985, J. Geophys. Res., 90, 163

Sheeley Jr., N. R., Walters, J. H., Wang, Y.-M., \& Howard, R. A. 1999, J. Geophys. Res., 104, 24739

Thernisien, A. F. R., Howard, R. A., \& Vourlidas, A. 2006, ApJ, 652, 763

Titov, V. S., \& Demoulin, P. 1999, A\&A, 351, 707

Tokman, M., \& Bellan, P. M. 2002, ApJ, 567, 1202

Török, T., \& Kliem, B. 2005, ApJ, 630, L97

Vršnak, B. 1990, Sol. Phys., 129, 295

Vršnak, B., Ruždjak, D., Sudar, D., \& Gopalswamy, N. 2004, A\&A, 423, 717

Vršnak, B., Sudar, D., \& Ruždjak, D. 2005, A\&A, 435, 1149

Xie, H., Offman, L., \& Lawrence, G. 2004, J. Geophys. Res., 109, A03109

Xue, X. H., Wang, C. B., \& Dou, X. K. 2005, J. Geophys. Res., 110, A08103

Yashiro, S., Gopalswamy, N., Michalek, G., et al. 2004, J. Geophys. Res., 109, A07105

Yeh, C.-T., Ding, M. D., \& Chen, P. F. 2005, Sol. Phys., 229, 313

Zhao, X. P., Plunkett, S. P., \& Liu, W. 2002, J. Geophys. Res., 107, SSH 13-1 